\title{
Spatial organization acts on cell signaling: how physical force contributes to the development of cancer
}

\author{
Marija Plodinec and Cora-Ann Schoenenberger*
}

\begin{abstract}
Cells constantly encounter physical forces and respond to neighbors and circulating factors by triggering intracellular signaling cascades that in turn affect their behavior. The mechanisms by which cells transduce mechanical signals to downstream biochemical changes are not well understood. In their work, Salaita and coworkers show that the spatial organization of cell surface receptors is crucial for mechanotransduction. Consequently, force modulation that disrupts the mechanochemical coupling may represent a critical step in cancerogenesis.
\end{abstract}

\section{Background}

The ability of cells to respond to mechanical signals from the environment plays an essential role in a myriad of biologically relevant functions such as cell migration [1], growth and differentiation [2]. In general, we can differentiate cellular responses to physical force into a purely mechanical response predominantly consisting of the cell's load-bearing deformation of cytoskeletal structures [3], and into biochemical signaling cascades where force propagation is relayed through membrane proteins or protein complexes to intracellular chemical signaling networks. Alterations in mechanotransduction often result in diseases such as cancer [4], arthritis [5] or atherosclerosis [6]. Resolving the mechanisms underlying mechanochemical coupling is therefore of fundamental importance.

One emerging mechanism through which mechanical forces may affect downstream signal transduction pathways involves the spatial organization of cell surface receptors [7]. A special case is that of juxtacrine

*Correspondence: Cora-ann.schoenenberger@unibas.ch

M.E.Müller Institute for Structural Biology, Biozentrum, University of Basel, Klingelbergstrasse 50/70, CH-4056 Basel/Switzerland interactions; for example, ephrin-A1 on one cell binds to EphA2 receptor tyrosine kinase on the apposed cell surface, which will induce assembly of higher-order clusters that trigger bidirectional signaling cascades in interacting cells [8,9]. Because EphA2 is overexpressed in $40 \%$ of mammary carcinomas and is functionally impaired in many other types of cancer [10], unveiling the mechanism by which the spatial organization of EphA2 receptors can affect the downstream cellular response to ephrin ligands is essential. Recent advances using nanolithography provide new insights into how the ephrinEph signaling system responds to different mechanical aspects of interacting cells [11]. These findings represent an important step towards understanding mechanochemical coupling and give us a glimpse into the significance of mechanical force in health and disease.

\section{Article}

In their recent study, Salaita and colleagues have established a procedure for investigating spatiomechanical concepts involved in the EphA2 signaling pathway [11]. The authors managed to reconstitute in vitro the juxtacrine signaling geometry between living cells expressing the EphA2 receptor tyrosine kinase and the laterally mobile ephrin-A1 ligand displayed on a fluid lipid bilayer supported on a glass substrate. Furthermore, by employing nanolithography the researchers were able to set physical barriers to the ligand mobility on the supported membrane. Their work shows that the mechanical ligand restriction extends to the spatial organization of EphA2 receptor at cell surface junctions and alters the cellular response to ephrin-A1.

Salaita and coworkers scrutinized two experimental conditions: one in which EphA2-expressing cells are interacting with ephrin-A1 ligand that has an unrestricted lateral mobility on a fully saturated lipid bilayer, and a second where ephrin is presented on a fluid membrane that is physically constrained by an underlying pattern of nanofabricated metal lines. In the first scenario, ephrin-A1-EphA2 interaction triggered spatial reorganization of the receptor on the cell membrane into 
microclusters that undergo inward radial transport. In contrast, when the cells expressing EphA2 receptors contact what the authors call spatial mutations, the receptor and associated signaling molecules became equally constrained as the boundaries impede radial transport of Eph-ephrin microclusters. Local receptor activation, however, occurred irrespective of the substrate geometry.

Total internal reflection microscopy tracking of unrestricted fluorescently labeled ephrin-A1 and green fluorescent protein-labeled actin revealed an annular association of F-actin with the EphA2 clusters. Moreover, actomyosin contractility was shown to be the driving force of radial cluster movement. Consistent with an association of F-actin with EphA2, restriction of receptor movement changed the cytoskeleton to a spread morphology with filamentous actin predominantly concentrated in lamellopodia at the cell periphery.

To establish whether the propensity to radially transport the EphA2 receptor can be used to characterize breast cancer cell lines, Salaita and colleagues determined a radial distribution function for 26 mammary cancer cell lines with different molecular and phenotypic signatures in neoplasia. The spatial organization phenotypes were then correlated with genomic and proteomic data available from these lines. There was no correlation to the mRNA and protein expression levels of EphA2; however, an association between radial EphA2 transport and signaling pathways that are associated with invasiveness - such as ErbB, p53, integrin and mitogenactivated protein kinase - became apparent. In addition, more aggressive cell lines exhibited larger complex clusters. The authors conclude that the spatial organization of the EphA2 receptor, which is modulated by mechanical aspects of the microenvironment, could serve as a marker for cancer progression.

\section{Viewpoint}

By mechanically restricting the movement of cell surface molecules, Salaita and coworkers have convincingly shown that external physical forces alone are sufficient to modify downstream cellular activities. In their system, the EphA2/ephrin-A1 complex acts as a force sensor that, by radial movement and molecular clustering, transduces mechanical signals from the environment to a chemical response of the cell. Notably, the signaling pathways affected upon force modulation are those that play a role in the onset and progression of cancer.

Specific physical parameters of the environment, such as the texture or geometry of the surrounding tissue, have previously been shown to be important phenotypic determinants of mammalian cells [12]. Given the sensitivity to mechanical restriction displayed by the EphA2/ ephrin-A1 signaling complexes, one could imagine that force measurements in general could be valuable markers for tumor characterization. Consistent with this notion, recent findings emphasize force being a key factor in cancer progression [13]. The kind and length scale of forces a cell needs to experience to first transform into a cancer cell and later on assume a more aggressive phenotype, however, remains an enigma.

In the near future we will hopefully learn about the fine-tuning of mechanical stimuli that promote a switch from the normal to the malignant phenotype and vice versa. Considering that the force-sensing mechanisms could be sites for therapeutic intervention, the relevance of gaining knowledge on mechanotransduction is more than obvious. Moreover, by resolving the downstream signaling pathways involved in a mechanically induced switch of phenotype, targets for anti-cancer therapeutic agents that can counteract the applied force might be identified. Possibly, drugs that are administered for other diseases could act on the mechanical coupling step as well.

In conclusion, linking mechanical aspects to biochemical approaches is a promising way to gain knowledge on tumorigenesis, and thus opens new avenues for cancer therapies in the future.

\section{Competing interests}

The authors declare that they have no competing interests.

\section{Acknowledgements}

The authors are supported by the National Center of Competence in Research Nanoscale Science, the Swiss National Science Foundation, and the M.E.

Müller Foundation.

\section{Published: 23 August 2010}

\section{References}

1. Janmey PA, McCulloch CA: Cell mechanics: integrating cell responses to mechanical stimuli. Annu Rev Biomed Eng 2007, 9:1-34

2. Discher DE, Janmey P, Wang YL: Tissue cells feel and respond to the stiffness of their substrate. Science 2005, 310:1139-1143.

3. Kasza KE, Rowat AC, Liu J, Angelini TE, Brangwynne CP, Koenderink GH, Weitz DA: The cell as a material. Curr Opin Cell Biol 2007, 19:101-107.

4. Paszek MJ, Zahir N, Johnson KR, Lakins JN, Rozenberg Gl, Gefen A, ReinhartKing CA, Margulies SS, Dembo M, Boettiger D, Hammer DA, Weaver VM: Tensional homeostasis and the malignant phenotype. Cancer Cell 2005, 8:241-254.

5. Millward-Sadler SJ, Wright MO, Lee H-S, Caldwell H, Nuki G, Salter DM: Altered electrophysiological responses to mechanical stimulation and abnormal signalling through $\alpha_{5} \beta_{1}$ integrin in chondrocytes from osteoarthritic cartilage. Osteoarthritis Cartilage 2000, 8:272-278.

6. Hahn C, Schwartz MA: The role of cellular adaptation to mechanical forces in atherosclerosis. Arterioscler Thromb Vasc Biol 2008, 28:2101-2107.

7. Shen K, Thomas VK, Dustin ML, Kam LC: Micropatterning of costimulatory ligands enhances CD4+ T cell function. Proc Natl Acad Sci U S A 2008, 105:7791-7796.

8. Qi Murai KK, Pasquale EB: 'Eph'ective signaling: forward, reverse and crosstalk. J. Cell Sci 2003, 116:2823-2832.

9. Marquardt T, Shirasaki R, Ghosh S, Andrews SE, Carter N, Hunter T, Pfaff SL: Coexpressed EphA receptors and ephrin-A ligands mediate opposing actions on growth cone navigation from distinct membrane domains. Cell 2005, 121:127-139

10. Zelinski DP, Zantek ND, Irizarry AR, Kinch MS: EphA2 overexpression causes tumorigenesis of mammary epithelial cells. Cancer Res 2001, 61:2301-2306.

11. Salaita K, Nair PM, Petit RS, Neve RM, Das D, Gray JW, Groves JT: Restriction of receptor movement alters cellular response: physical force sensing by 
EphA2. Science 2010, 327:1380-1385.

12. Vogel V, Sheetz M: Local force and geometry sensing regulate cell functions. Nat Rev Mol Cell Biol 2006, 7:265-275.

13. Kumar S, Weaver VM: Mechanics, malignancy, and metastasis: the force journey of a tumor cell. Cancer Metastasis Rev 2009, 28:113-127. doi:10.1186/bcr2623

Cite this article as: Plodinec M, Schoenenberger C-A: Spatial organization acts on cell signaling: how physical force contributes to the development of cancer. Breast Cancer Research 2010, 12:308. 